\title{
Correspondence
}

TO THE EDITOR, British Journal of Venereal Diseases

Sir,

\section{Pityriasis versicolor of the glans penis}

Pityriasis versicolor is a common disease, more so in tropical than in temperate climates.

An Indian man, aged 20, employed as a cook's assistant in the government hospital, attended the dermatology and venereology clinic complaining of loss of pigmentation and scaly patches on his body and tip of his penis. The condition had first developed three years previously with lesions on the upper trunk. He had never taken any treatment but became alarmed when the condition spread progressively and appeared on the genitals, occasionally causing irritation. His general health was good and he gave no past or family history of notable illnesses.

On examination there was a depigmented area on the chest, back, and buttocks formed by coalescence of smaller areas of pityriasis versicolor, which were present elsewhere including the shaft of the penis. The patient had been circumcised in childhood and on the left side of the glans penis there were two circinate, discrete areas of fine scaling, slightly raised, the smaller being on the rim of the glans. These lesions were unlike any other forms of balanitis which we had seen.

Radiation with Wood's light showed yellow fluorescence of some of the lesions on the trunk and all of those on the glans and shaft of the penis. With flashlight photography of the lesions under Wood's light the yellow fluorescence was lost but the glandular lesions showed as areas of scaly white. Scrapings from both trunk and glans gave positive results for spores and mycelium typical of pityriasis versicolor.

The patient was treated with twice-daily application of miconazole cream, and the glandular lesion had resolved to hypopigmentation when he was seer: a week later. He did not attend again.

Four hundred cases of pityriasis versicolor were diagnosed out of a total of more than 8000 new referrals in a six-month period, which gives an incidence of approximately $5 \%$. In hot climates involvement of the skin is frequently extensive but the genitals are less commonly affected. Total body examination with Wood's light is the only means of determining the extent of the disease in dark-skinned patients, and it shows areas of which the patient may be unaware. Five cases of pityriasis versicolor affecting the penile skin were diagnosed among 70 men during a six-month series here. The patient described above had lesions on the skin and on the glans, and this association has not been reported before, even in such detailed studies as that of Roberts (1968) or El-Hefnawi (1971).

It is interesting that pityriasis versicolor does not affect the genitals more, considering the role that heat and moisture are considered to play in its aetiology. On the other hand, it is easy to understand why the surface of the glans is unaffected, because of its different structure.

\section{Yours faithfully,}

\section{A. Karim Nia}

E. L. Smith

Department of Dermatology,

Government Hospital,

Abu Dhabi,

United Arab Emirates

\section{References}

El-Hefnawi, H., El-Gothamy, Z., and Refai, M. (1971). Studies in pityriasis versicolor in Egypt. Mykosen, 14 (5), 223-231.

Roberts, S. O. B. (1968). Pityriasis versicolor: a clinical and mycological investigation. British Journal of Dermatology, 81, 315-326.

Smith, E. L. (1978). Pityriasis versicolor of the penis. British Journal of Venereal Diseases, 54, 441 .

TO THE EDITOR, British Journal of Venereal Diseases

Sir,

\section{Anal canal versus rectal wall sampling}

Dr R. S. Morton's criticism of my paper (Morton, 1978) has surprised me. It is not that I feel I am beyond criticism, but it is the totally uncalled for manner in which he has done so.

To add insult to injury he has even made incorrect statements. Even the title of my paper has not been correctly interpreted; my paper was entitled 'Diagnosis of rectal gonorrhoea by blind anorectal swabs compared with direct vision swabs taken via a proctoscope'. He has changed this to
'Anal canal versus rectal wall sampling'; it appears to me that he does not understand the word anorectal.

May I point out to Dr Morton once again what I was attempting to prove. The study I did was to compare the results of gonococcal cultures taken from the same site, namely the rectum, using two different methods for obtaining the necessary $\overrightarrow{\vec{\omega}}$ material. I used the words 'blind anorectal swab' because it is not possible to pass a के blind swab into the rectum without first passing it through the anal canal. I con- or cluded from this study that both methods $\omega$ gave satisfactory culture results.

This being the conclusion, what I would like to know from Dr Morton is how more cases of gonococcal proctitis could be diagnosed if a proctoscope had been used. Another misquotation is, "he reached the conclusion that rectal wall sampling should be confined to the first visits and to patients with symptoms'. What I wrote was, 'It is, however, not suggested that proctoscopic examination should not be done at all but that it could be confined to the first visit and to patients who complain of symptoms'. The word 'should' cannot be substituted for the word 'could' without changing the meaning.

Is he really serious when he says, 3 'Vaginal sampling is not the best way to diagnose gonococcal endocervicitis'? Is this not obvious? It is certainly not advisable to take a swab from one site to make a diagnosis of an infection at another site; if one does this the results are likely to be unsatisfactory. Despite Dr Morton's 8 implication, this is not what I did.

Finally, it was notable that Dr Morton did not quote any figures to support his argument.

Yours faithfully, P. Deheragoda N

Department of Genitourinary Medicine,

Croydon General Hospital,

London Road,

Croydon,

Surrey CR9 2RH

References

Deheragoda, P. (1977). Diagnosis of rectal gonorrhoea by blind anorectal swabs compared $\square$ with direct vision swabs taken via a proctoscope. British Journal of Venereal Diseases, 53, 311313.

Morton, R. S. (1978). Anal canal versus rectal wall $\overparen{D}$ sampling. British Journal of Venereal Diseases, 54. 206. 